\title{
The modelling of condensation in horizontal tubes and the comparison with experimental data
}

\author{
Rafat Bryk ${ }^{1, *}$, Holger Schmidt ${ }^{2}$, Thomas Mull ${ }^{2}$, Thomas Wagner ${ }^{2}$, Oliver Herbst ${ }^{2}$, and Ingo Ganzmann ${ }^{2}$ \\ ${ }^{1}$ Warsaw University of Technology, Institute of Heat Engineering, 00-661 Warsaw, Poland \\ ${ }^{2}$ AREVA GmbH, Thermal Hydraulics and Components Testing, 91052 Erlangen, Germany
}

\begin{abstract}
The condensation in horizontal tubes plays an important role in determining the operation mode of passive safety systems of modern nuclear power plants. In this paper, two different approaches for modelling of this phenomenon are compared and verified against experimental data. The first approach is based on the flow regime map developed by Tandon. Depending on the regime, the heat transfer coefficient is calculated according to corresponding semi-empirical correlation. The second approach uses a general, fully empirical correlation proposed by Shah. Both models are developed with utilization of the object-oriented, equation-based Modelica language and the open-source OpenModelica environment. The results are compared with data obtained during a large scale integral test, simulating a Loss of Coolant Accident scenario performed at the dedicated Integral Test Facility Karlstein (INKA) which was built at the Components Testing Department of AREVA in Karlstein, Germany. The INKA facility was designed to test the performance of the passive safety systems of KERENA, the new AREVA boiling water reactor design. INKA represents the KERENA containment with a volume scaling of 1:24. Components heights and levels over the ground are in the full scale. The comparison of simulations results shows a good agreement.
\end{abstract}

\section{Introduction}

KERENA is a medium-capacity boiling water reactor developed by AREVA GmbH [1]. It utilizes several innovative passive safety systems that ensure safe depressurization and heat removal in case of an emergency or accidents, including a loss of coolant accident (LOCA). The main components of KERENA passive safety features are a Containment Cooling Condenser (CCC), Emergency Condenser (EC), Passive Pressure Pulse Transmitters (PPPT) and a Passive Core Flooding System. These systems utilize basic physical laws such as gravity and free convection. Therefore, in order to operate them, there is no need for an external power supply and additional devices such as pumps. This in turn leads to a significant reduction of costs during construction of the plant.

In order to examine the performance of the passive systems, a dedicated test facility was built at the Components Testing Department of AREVA in Karlstein, Germany. The Integral Test Facility Karlstein (INKA) represents the KERENA containment with a volume scaling of 1:24. Components heights and levels over the ground are kept in the full scale. The test rig setup allows us to investigate each component individually as well as the entire system during integral tests. For the purposes of testing the integral system, INKA is equipped with several large vessels corresponding to individual pools and vessels of KERENA. The steam accumulator of the
Karlstein large valve test facility GAP represents the Reactor Pressure Vessel (RPV). The vessel has a storage capacity of $1 / 6$ of the KERENA RPV. In order to simulate the decay heat of the core, the vessel is fed with steam by a Benson boiler with maximum power output of $22 \mathrm{MW}$. The vessel is designed for operational pressure up to 160 bars so experiments may be performed starting from the operation point of boiling water reactor of 70 bars.

Compartments of the KERENA RPV are represented by three separate vessels: Flooding Pool Vessel, Pressure Suppression Pool Vessel and Drywell Vessel. An additional tank is used as a representation of Shielding/Storage Pool Vessel.

Due to the appropriate instrumentation concept and the density of sensors, data obtained during experiments performed at the facility may be utilized for validation of thermal-hydraulic codes. Therefore, measurements collected during one of the tests performed in the framework of the Integral Experimental and Analytical Safety Cases for Design-Basis Accidents Featuring Passive Systems were used to validate condensation models developed in open source OpenModelica Connection Editor environment utilizing Modelica language.

Modelica is a non-proprietary, object-oriented, equation-based language to conveniently model complex physical systems [2]. Several environments utilize the language. Models described in this paper were developed with OpenModelica Connection Editor - an open-source

\footnotetext{
*orresponding author: test-labs@areva.com
} 
environment developed by Open Source Modelica Consortium for industrial and academic usage [3]. Modelica is equipped with a large multi-domain, opensource library. Components encapsulated in the library may be developed; enhanced and other objects may inherit their features.

\section{Operation of KERENA Emergency Condenser}

The two main purposes of the Emergency Condenser are heat removal and depressurization of the RPV in abnormal conditions. The principle of the EC operation is presented in Fig. 1.

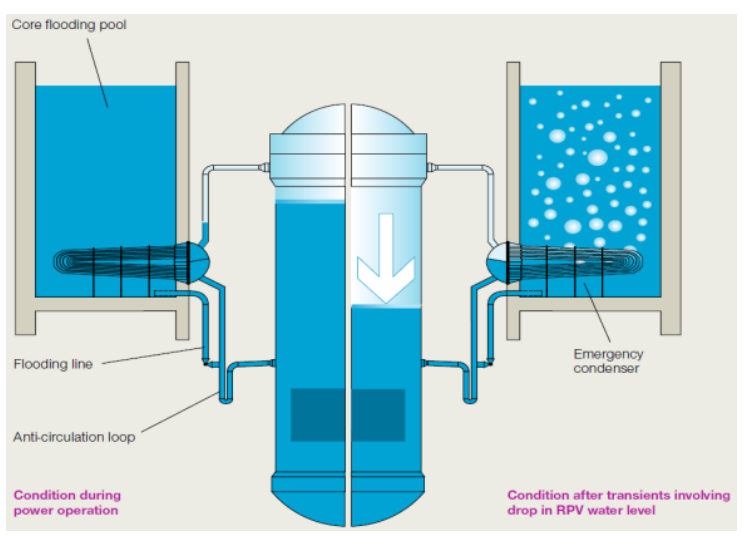

Fig. 1. Operation scenarios of the Emergency Condenser.

The EC is composed of a large number of slightly inclined tubes. The tubes are submerged in the Core Flooding Pool and they are filled with water during regular operation of the plant. Should the water level in the RPV decrease, the water drains from the tubes and the steam from the RPV enters the EC tubes. Due to the contact of the tube walls with the cold water at the secondary side in the Core Flooding Pool heat transfer occurs, leading to condensation of the steam in the tubes. Since the tubes of the Emergency Condenser are inclined slightly, the condensate flows back to the RPV due to gravity. The Emergency Condenser is connected to the RPV without any isolating elements and it comes into action automatically without any need for electrical power. Thus, the device is operated according to the natural law of communicating vessels.

\section{Models development}

\subsection{System description and governing equations}

The analysed thermal-hydraulic system was a straight pipe slightly inclined to horizontal orientation. The flow is governed by the mass, energy and momentum conservation equations, as 1-dimensional, partial differential equations, to which the following assumptions are applied:

- uniform velocity is assumed on cross sections,

- longitudinal heat diffusion term is neglected,

- accumulation is considered in each volume,

- influence of the angulation of the EC pipes is neglected,
- thermal-hydraulic properties are calculated on the basis of pressure and enthalpy at each node,

- boundary conditions are assumed according to the measurements,

- temperature at the external wall of the tube is taken from measurements,

- tube wall is assumed to have constant thermal conductivity,

- heat transfer coefficient at the secondary side is neglected,

- tube length and wall thickness are modelled according to EC specification

- tube bundle influence is not taken into account.

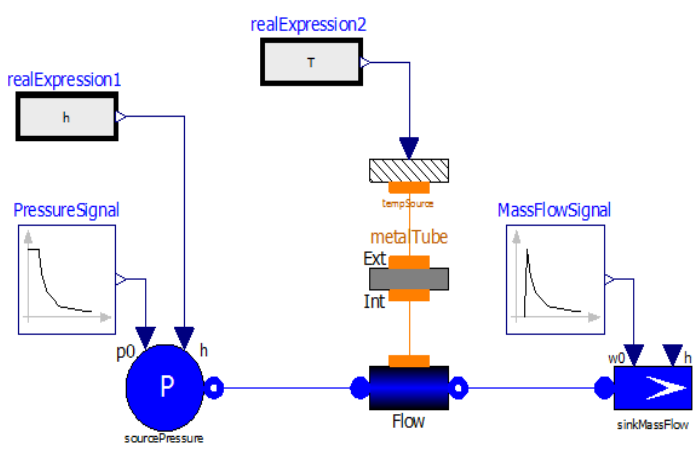

Fig. 2. Object-oriented configuration of the model.

The model was set up by adopting an extended version of the Flow1DFV2ph component of the Modelica ThermoPower library. The component, in which the balance equations are discretized by means of the finite volume approach, was modified by formulation of momentum balance equation for each volume instead of the whole component. This leads to a more detailed description by enabling the calculation of fluid properties and saturation conditions at each node, taking into account pressure in the particular node. Before modification, fluid properties at each node were evaluated either with respect to inlet or outlet pressure.

The mass balance equation in each volume is given by:

$$
\dot{m}_{j}-\dot{m}_{j+1}=A l \frac{d \rho_{k}}{d t}
$$

Taking pressure and specific enthalpy as state variables:

$$
\dot{m}_{j}-\dot{m}_{j+1}=A l\left[\left(\frac{\partial \rho_{k}}{\partial h_{k}}\right)_{p} * \frac{d h_{k}}{d t}+\left(\frac{\partial \rho_{k}}{\partial p_{j}}\right)_{h} * \frac{d p_{j}}{d t}\right]
$$

The energy balance equation in each volume is given by:

$$
\dot{m}_{j} * h_{j}-\dot{m}_{j+1} * h_{j+1}+Q_{k}=\operatorname{Al}\left(\rho_{k} \frac{d h_{k}}{d t}-\frac{d p_{k}}{d t}\right)
$$

The momentum balance equation for each volume is given by:

$$
p_{j}-p_{j+1}=\frac{l}{A} * \frac{d w_{k}}{d t}+\rho_{k} g l \sin \theta+\Delta p_{k, f}+\Delta p_{k, a}
$$

with acceleration and friction pressure losses for each volume given by: 


$$
\begin{gathered}
\Delta p_{k, a}=\frac{1}{A^{2}} * w_{k}^{2} *\left(\frac{1}{\rho_{j+1}}-\frac{1}{\rho_{j}}\right) \\
\Delta p_{k, f}=\zeta \frac{2 \Lambda_{k} l}{D A^{2} \rho_{k}} * w_{k}^{2}
\end{gathered}
$$

Where $\Lambda_{\mathrm{k}}$ is calculated with Colebrook correlation:

$$
\Lambda_{k}=\frac{0.332}{\ln \left(\frac{e}{3.5}+\frac{5.47}{R e_{k}^{0.9}}\right)^{2}}
$$

In equations, 1-7 $\mathrm{A}$ and 1 stand respectively for tube cross section length of a single volume. Subscript K denotes volume number while $\mathbf{J}$ stands for the number of the nodes. Subscripts $\mathrm{p}$ and $\mathrm{h}$ mean derivatives with constant pressure and enthalpy respectively.

In the flow model, a replaceable module for heat transfer calculation was introduced. This allowed calling different models of heat transfer developed as separate objects.

Regarding the modelling of the heat exchange, new heat transfer components were introduced in order to implement correlations expressing two approaches described in subsections 3.2 and 3.3. These models were then called in the flow model by redeclare model clause in the text view of the whole system model presented as diagrams in fig. 2.

\subsection{Flow regime map based model}

The flow regime map based model was developed utilizing the map proposed by Tandon [8]. The model calculates the actual heat transfer coefficient by switching between several flow regimes dependent on semiempirical correlations. Fig. 3 illustrates different flow patterns in horizontal tubes. The flow regime map, together with correlations applied in specific regime is shown in Fig. 4.

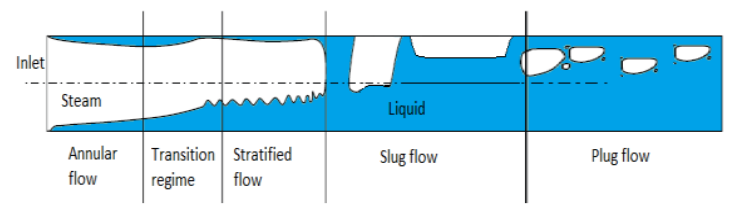

Fig. 3. Flow patterns during condensation in horizontal tube [4].

Tandon's flow regime map is based on the volume ratio of liquid and gas in a cross section area $(1-\varepsilon) / \varepsilon$, where $\varepsilon$ stands for the void fraction, and on the dimensionless steam velocity:

$$
j_{D}^{*}=\frac{x G}{\left[g D \rho_{V}\left(\rho_{L}-\rho_{V}\right)\right]^{0.5}}
$$

Since the EC pipes are initially filled with liquid water and due to possible single-phase condensate flow at the lower sections of the tube, an additional correlation for single phase liquid water flow was implemented into the model. Table 1 indicates all correlations and application ranges.

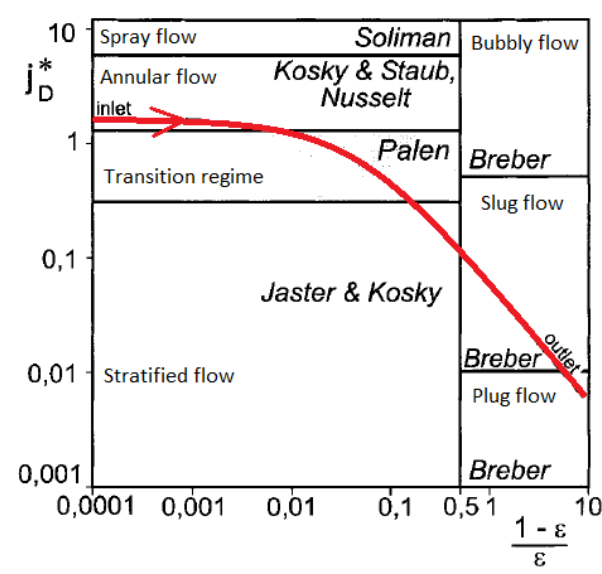

Fig. 4. Tandon's flow regime map [4].

In the table subscripts, Fi and $\mathrm{V}$ indicate whether a variable refers to condensate film or vapour. Further details of different pattern formation development of the correlations can be found in [8-14].

\subsection{General correlation model}

The implemented general correlation for condensation in horizontal tubes was developed by Shah [16]:

$$
\begin{gathered}
\alpha=\alpha_{S P h}\left[(1-x)^{0.8}+\frac{3.8 * x^{0.76} *(1-x)^{0.04}}{p_{r}^{0.38}}\right] \\
\alpha_{S P h}=0.023 R e_{L}^{0.8} \operatorname{Pr}_{L}^{0.4} \frac{\lambda}{D}
\end{gathered}
$$

The correlation was developed by the analysis with a wide variety of experimental data. This includes different fluids and pipe orientation. Details of development of the correlation may be found in [16].

\section{Results}

Experimental data for model validation was obtained during the simulation of the Main Steam Line Break (MSLB), taking into account heat decay. The test was performed at the INKA test facility in the framework of the EASY Project. Since the MSLB experiment was conducted in order to investigate performance of the integral system rather than a single component, it should be emphasised that other components like Reactor Pressure Vessel, Drywell Vessel, Pressure Suppression Pool, Shielding/Storage Pool and Standpipe strongly influenced the behaviour of the Emergency Condenser. Furthermore, the test course assumed certain activities at certain points, which lead to strong transients and backflows. This can be seen in Fig. 5, where measured flows are plotted together with mass flow applied to the models. The red line, representing the inlet mass flow, is clearly delayed with respect to outflow measurement. Paradoxically, thermocouples inside EC tubes indicate an increase in water temperature (fig. 7) before steam enters the tubes according to Fig. 5. 
Table 1. Heat transfer correlations with respect to flow regime.

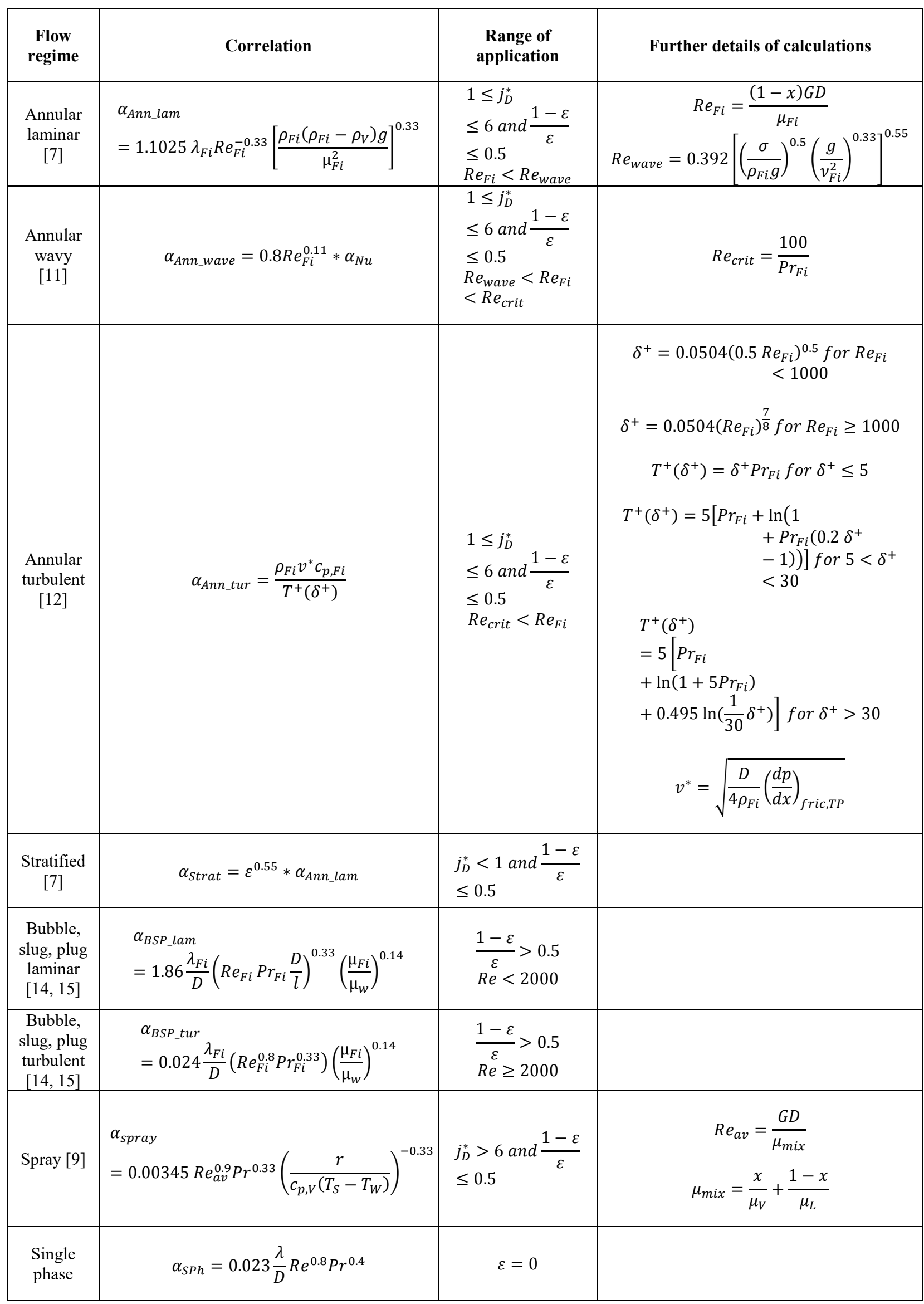


This means that steam enters the EC tubes earlier than indicated in Fig. 4, but pressure transients resulted in negative mass flow at the inlet, which is presented as zero flow by the red line between 350 and 425 seconds of the test.

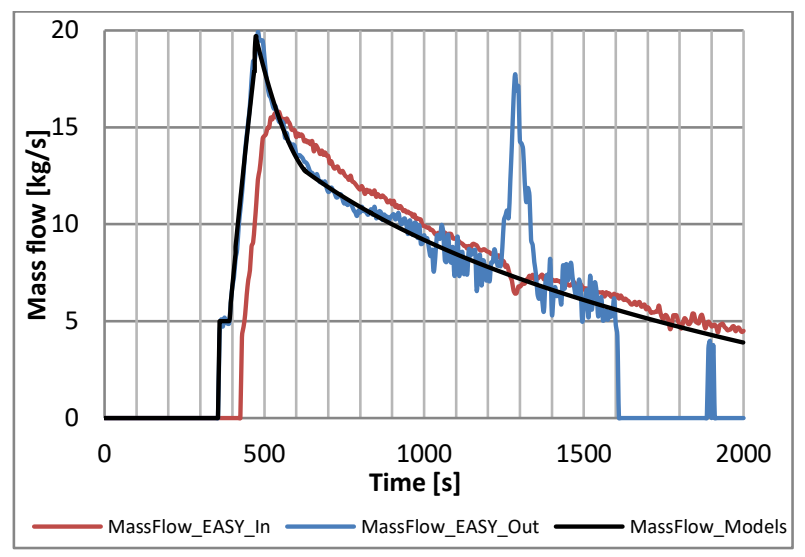

Fig. 5. Measured and modelled mass flows.

Similarly, the measured outflow peak after 1200 seconds and zero-flow after 1500 are caused by the performance of other devices. These cannot be taken into account during validation of condensation model. Therefore, only a part of the test was considered for further analysis. The time taken into account was between 0 and 1200 seconds of the test. This covers the initial operation of the system and the response of the EC to the fast transient caused by steam line break and corresponding RPV water level decrease. The break was initiated 350 seconds after the beginning of the test. At this time, steam from the RPV was released into the DWV, leading to a decrease of pressure in the RPV. This in turn resulted in RPV water level decreasing and forced the flow from the standpipe and EC into the RPV.

The mass flow between EC and RPV enabled the steam to enter the EC tubes (as presented on the right side of fig.1). The contact of saturated steam with cold tube walls leads to heat exchange and condensation of steam. The power of the EC calculated on the basis of measurements is presented in the figure below together with these computed by the models. The dashed lines indicate $+/-15 \%$ deviations from the measurement curve.

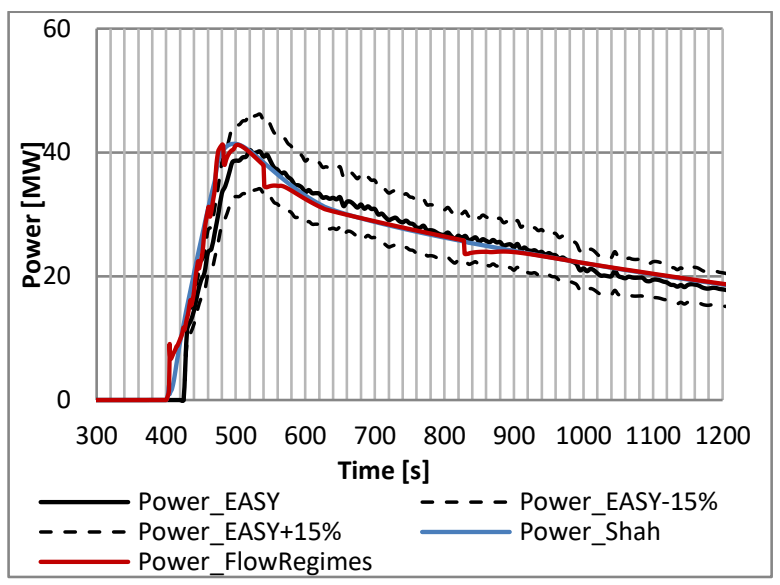

Fig. 6. Heat flow rates according to measurements and models.
Fig. 6 shows a good agreement between heat flow rate obtained from measurements and these computed by the models. Although both curves plotted according to simulations results have similar shapes, analysis of them clearly indicates their different origins. The blue line has a smooth shape, since power is calculated based on heat transfer coefficient correlation. Peaks of the red line result from switching to different correlations depending on transient flow regime. These peaks are even more apparent during analysis of single volumes. Nevertheless, both models provide satisfactory agreement.

Regarding the fluid temperature, two points were chosen to compare measurements against calculations. The first measurement point is situated before the angulation of the EC pipes i.e. at the $\mathrm{x}=4.423 \mathrm{~m}$, where $\mathrm{x}$ is the longitudinal coordinate of the pipe. Corresponding calculation point was $\mathrm{x}=4.32 \mathrm{~m}$. The discrepancy of around $10 \mathrm{~cm}$ is caused by the location of thermocouples during the test and nodalization of the models. The second measurement point is located at $x=5.756 \mathrm{~m}$ i.e. at the bottom part of angulation of the pipe. The picture below shows temperatures measured at both locations and calculated values at corresponding positions. Solid lines represent the first point while dashed lines demonstrate fluid temperature at second point.

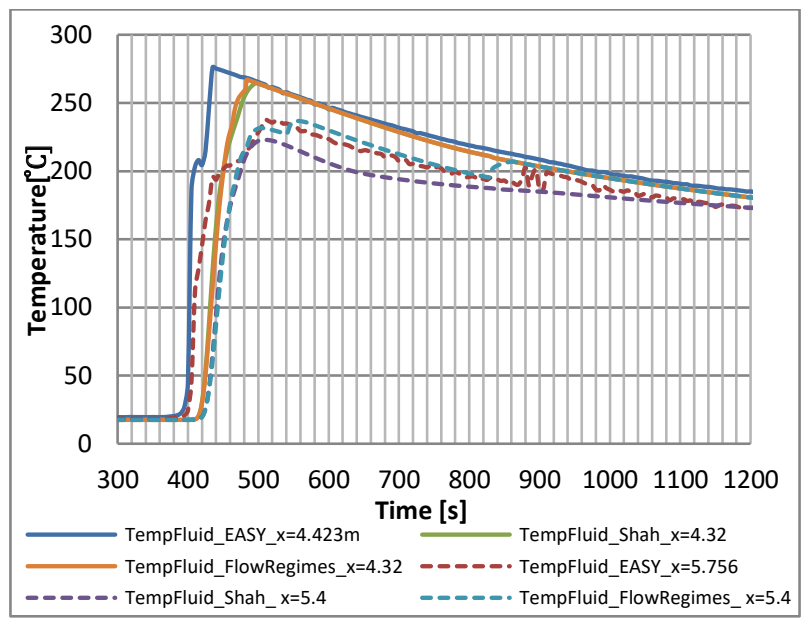

Fig. 7. Fluid temperatures.

Solid lines in Fig. 7 indicate that around 4.3 meters from the tubes inlet the flow is still a two-phase mixture at the saturation temperature. Fast transients of pressure and mass flows cause the delay of the temperature increase after initialization of the break. The dashed lines demonstrate that at the second position, the flow is already subcooled. The time delays of the transient in both models are still present, but it can be noticed that the flow regimes based model evaluates the temperature more accurately.

Considering the wall temperature, due to the falling water film and stratification, significantly different values were measured at the upper and bottom wall of the tube. Therefore, two measurements are presented in the chart below. Both come from the part of EC before the angulation, one represents the upper part of the wall, and the second the bottom part. Since the water film at the bottom part of the tube is thicker, the heat transfer at this 
part of the tube is lower, and so is the temperature. The figure below shows measured and calculated values.

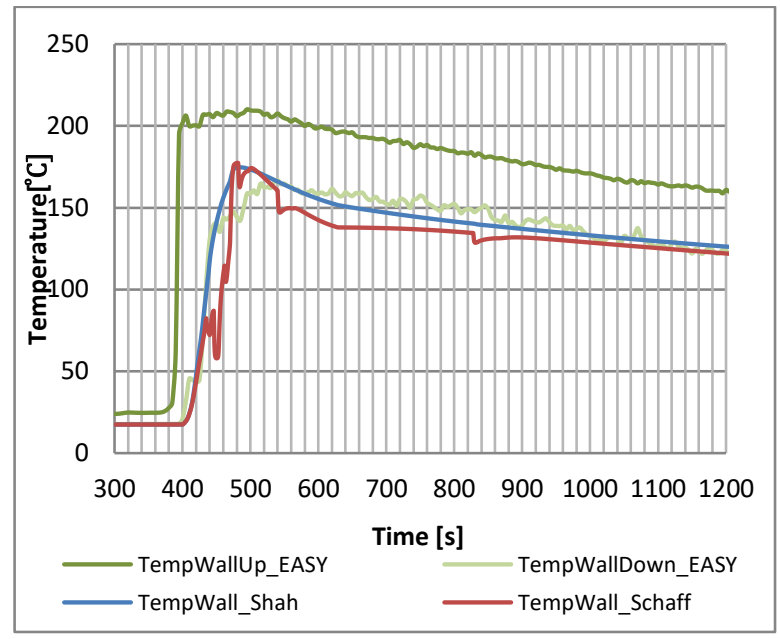

Fig. 8. Wall temperatures.

Since the wall temperature in both models was calculated without distinction of the top and bottom side, there is a significant discrepancy between measured and calculated values. Wall temperature calculated by the models is much better to evaluate than the one measured at the bottom part, while the measured one at the top part is around $40^{\circ} \mathrm{C}$ higher. This significant discrepancy will be the subject of future work, which will focus on modelling of condensation with taking into account falling film angle around the top of the tube.

\section{Conclusions and outlook}

Two different approaches for modelling of condensation in horizontal tubes have been investigated and implemented, with utilization of object-oriented Modelica language and OpenModelica environment. The aim of this work was investigation, verification and comparison of the performance of models applied with usage of opensource OpenModelica environment in order to draw conclusions considering their reliability in modelling of passive safety systems. The main conclusions are:

- both models predict heat flow rate with satisfactory accuracy,

- flow regime based model evaluates fluid temperature more accurately (fig. 7),
- since the flow regime based model switches between different heat transfer coefficient correlations, significant peaks of power and temperature were observed,

- these peaks may lead to discrepancies of parameters (wall temperature, fig. 8) and instabilities of simulations,

- due to thinner film layer at the upper wall part of the tube, the wall temperature is significantly higher which is not taken into account in the models,

- both models indicate correctly the point in which singlephase liquid water flow occurs.

\section{References}

1. R. Drescher, T. Wagner, S. Leyer, VGB PowerTech, 5, 33-37 (2014)

2. https://modelica.org/

3. https://openmodelica.org/

4. A. Schaffrath, E.F. Hicken, H. Jaegers, H.-M. Prasser, Nuc. Eng. and Des. 188, 303-318 (1999)

5. A. Schaffrath, A. Krüssenberg, A. Fjodorow, U. Gocht, W. Lischke, Nuc. Eng. and Des. 204, 251-265 (2001)

6. Verein Deutscher Ingenieure, VDI Heat Atlas, Second Edition, Springer-Verlag Berlin Heidelberg (2010)

7. W. Nusselt, Zeitschr. Vereins Deutscher Ingenieure 27, 541-546 and 569-575 (1961)

8. T.N. Tandon, H.K. Varma, C.P. Grupta, J. Heat Transfer 104, 763-768 (1982)

9. E.N. Soliman, Int. J. Multi-Phase Flow 12, 277-288 (1986)

10. S.S. Grimley, Trans. Inst. Chem. Eng. 23, 229-235 (1945)

11. S.S. Kutateladse, Fundamentals of Heat Transfer, Edward Arnold (1963)

12. P.G. Kosky, W.F. Staub, AICHE J. 17, 1037-1043 (1971)

13. H. Jaster, P.G. Kosky, Int. J. Heat Mass Transfer 19 , 95-99 (1976)

14. G.W. Breber, J.W. Palen, J. Taborek, Trans. ASME J. Heat Transfer 102, 471-476 (1980)

15. E.N. Sieder, G.E. Tate, Ind. Eng. Chem. 28, 14291435 (1936)

16. M.M. Shah, Int. J. Heat Mass Transfer 22, 547-556 (1979) 